\title{
Politique
}

\section{La presse et la politique municipale dans deux villes du Québec}

\section{André Blais et Jean Crête}

Volume 1, numéro 2, automne 1982

Les médias et les pouvoirs

URI : https://id.erudit.org/iderudit/040401ar

DOI : https://doi.org/10.7202/040401ar

Aller au sommaire du numéro

Éditeur(s)

Société québécoise de science politique

ISSN

0711-608X (numérique)

Découvrir la revue

Citer cet article

Blais, A. \& Crête, J. (1982). La presse et la politique municipale dans deux villes du Québec. Politique, 1(2), 41-67. https://doi.org/10.7202/040401ar d'utilisation que vous pouvez consulter en ligne.

https://apropos.erudit.org/fr/usagers/politique-dutilisation/ 


\title{
La presse et la politique municipale dans deux villes du Québec
}

\author{
André Blais \\ Université de Montréal \\ Jean Crête \\ Université Laval
}

Le thème «Les médias et les pouvoirs » attire l'attention sur un phénomène particulier d'information et de communication dans nos sociétés contemporaines et dans le jeu politique tout spécialement. Étant donné la place importante qu'occupent les médias dans ce jeu, il convient de bien situer leur fonction spécifique.

On peut concevoir l'information politique comme un jeu mettant en présence deux acteurs principaux: le gouvernement d'une part, la presse d'autre part (d'autres acteurs, les consommateurs de la presse en particulier, ne seront pas considérés ici). Les messages politiques contenus dans la presse traitent directement ou indirectement de ce que fait ou ne fait pas le gouvernement. Ces messages proviennent souvent des gouvernements. La presse peut les retransmettre ou non, les filtrer, les déformer ou ajouter d'autres éléments d'information. La presse et le gouvernement se retrouvent donc dans une situation d'interdépendance réciproque. Le gouvernement a besoin de la 
presse pour faire passer ses messages et celle-ci a besoin du gouvernement pour diffuser la nouvelle ${ }^{1}$.

Cette situation d'interdépendance soulève toute une série de questions. La première et la plus importante concerne la marge de manœuvre dévolue à la presse dans le processus d'information. La presse se contente-t-elle de retransmettre les messages fournis par le gouvernement ou joue-t-elle un rôle plus actif en filtrant ou en générant d'autres «nouvelles»? En autant que ce rôle n'est pas totalement passif, comment se fait ce filtrage? Certaines recherches ont suggéré que la presse privilégie les informations de nature polémique et négligent celles de nature administrative ${ }^{2}$. Qu'en est-il exactement? On peut d'autre part s'interroger à propos de l'impact de la presse sur le gouvernement. La presse exerce-t-elle une influence sur les décisions prises par le gouvernement, soit en réclamant ou dénonçant certaines actions, ou encore en dirigeant l'attention sur certains sujets précis ${ }^{3}$ ? C'est là une possibilité qu'il importe d'examiner, même si les cas d'influence directe semblent difficiles à démontrer ${ }^{4}$.

Notre étude porte sur le rôle de la presse dans deux villes du Québec, Sherbrooke et Trois-Rivières. Ce choix découle d'un certain nombre de considérations. D'une part, la recherche existante a tendance à négliger la presse régionale et locale, orientation qui a plusieurs conséquences malheureuses. Il en résulte un tableau passablement inexact de la réalité des médias;

1. Marcel Gilbert, «L'information gouvernementale et les courriéristes parlementaires au Québec», Revue canadienne de science politique, 4 (mars 1971): 26-52.

2. Gilbert, op. cit.; Hélène Cantin, La Tribune de la Presse au Parlement de Québec, Paris, Thèse de doctorat, Université de droit, d'économie et de sciences sociales, 1981, chap. 5 .

3. James C. Strouse, The Mass Media, Public Opinion and Public Policy Analysis: Linkage Explorations (Columbus, Charles E. Merrill, 1975).

4. Fred Fletcher, avec la collaboration de David V.J. Bell, André Blais, Jean Crête et William O. Gilsdorf, Les quotidiens et les affaires publiques (Ottawa, Commission royale sur les quotidiens, 1981). 
par exemple, on oublie souvent que le nombre de journaux locaux a augmenté sensiblement au cours de la décennie $70^{5}$. Certains indices suggèrent de plus que c'est au niveau municipal que l'impact de la presse peut être particulièrement prononcé ${ }^{6}$. D'autre part, nous voulions accorder une plus grande attention à la presse écrite. Or il appert que c'est au niveau de la nouvelle locale que le journal est utilisé comme source d'information privilégiée ${ }^{7}$. Nous avons cru bon, finalement, de ne pas nous limiter à une seule municipalité, de crainte de tomber sur un cas trop exceptionnel. Sherbrooke et Trois-Rivières ont été choisies parmi les quatre villes au Québec (à l'extérieur de Montréal et Québec) qui comptent un journal quotidien.

Dans les deux municipalités, le journal local appartient au groupe de presse contrôlé par GESCA. La Tribune de Sherbrooke compte environ 20 journalistes et son tirage varie autour de 40,000 alors que le Nouvelliste, de Trois-Rivières tire à environ 50,000 exemplaires et compte une quinzaine de journalistes. Dans chaque cas s'ajoutent deux éditorialistes. Un journaliste est assigné en permanence aux affaires municipales. D'autres journalistes peuvent traiter de dossiers particuliers. La page trois du journal est consacrée presque entièrement aux affaires municipales; dans le Nouvelliste, un peu plus de la moitié des articles de cette page touche la municipalité de Trois-Rivières, le reste traitant de deux municipalités voisines, alors qu'à la Tribune, -la même page est dévolue à Sherbrooke uniquement.

Sherbrooke compte également deux stations de télévision et cinq stations de radio et Trois-Rivières, une station de télé-

5. Ibid., 29.

6. André Blais et Jean Crête, «La presse et les affaires publiques au Québec», (étude, 1981, archives publiques).

7. Les mass media, Rapport du Comité spécial du Sénat sur les moyens de communication de masse, Ottawa, Imprimeur de la Reine, vol. III, 1970, 47; Gérald L. Grotta et al., "How Readers Perceive and Use a Small Daily Newspaper », Journalism Quarterly, 52 (automne 1975): 711-716. 
vision et quatres stations de radio. Les salles de nouvelles de ces médias électroniques sont beaucoup plus réduites et ne dépassent guère cinq journalistes. Dans aucun cas $y$ a-t-il un journaliste affecté exclusivement aux affaires municipales. Dans les deux municipalités, on retrouve aussi un hebdomadaire distribué gratuitement à la maison. En somme, dans les deux municipalités, le journal quotidien est celui qui consacre le plus de ressources à la couverture des affaires municipales, il est le seul medium à affecter un journaliste à plein temps à ce secteur. La radio, en particulier, reprend régulièrement les nouvelles originant du journal.

Notre recherche repose sur deux sources d'information. La première est une analyse de contenu du journal dans les deux municipalités, pour la période du $1^{\text {er }}$ octobre 1978 au 31 décembre 1980. Nous avons commencé en octobre 1978 de façon à couvrir l'élection municipale tenue au mois de novembre 1978. La deuxième est une série d'entrevues avec des informateurs privilégiés, soit les membres des conseils municipaux, des fonctionnaires, dirigeants d'associations, journalistes et cadres du journal. $\mathrm{Au}$ total, nous avons obtenu cinquante entrevues à Sherbrooke et trente-et-une à Trois-Rivières.

Il convient dès le départ de souligner les limites de notre étude. Considérons d'abord l'analyse de contenu. Nous avons étudié l'output du journal dont nous pouvons décrire plusieurs caractéristiques. Par contre, presque toutes les informations (nous en avons quelques-unes par le biais des entrevues) dont nous disposons sur l'administration municipale (décisions, règlements, services) proviennent du journal même. Il aurait été utile d'avoir des données indépendantes, avec lesquelles nous aurions pu confronter l'information véhiculée par le journal. De même, les entrevues ont porté sur les perceptions et opinions des informateurs; leurs réponses ne sauraient être interprétées comme correspondant automatiquement à la réalité. 


\section{La transmission de l'information gouvernementale}

Nous chercherons dans un premier temps à faire ressortir les principales caractéristiques du traitement que donne la presse des décisions et actions du gouvernement municipal. Nous voulons surtout dégager les grands traits de la démarche journalistique. Il s'agit en particulier de déterminer si la presse n'est qu'une courroie de transmission de l'information originant de l'administration municipale ou si elle joue un rôle plus actif dans la génération de nouvelles. Nous nous intéressons aussi à l'intérêt que la presse accorde à la controverse. L'on connaît l'attrait de la presse nationale pour la polémique ${ }^{8}$. Au niveau local, la situation est ambiguë. Cet attrait est tempéré par une plus grande valorisation de l'esprit civique qui peut amener à penser davantage en fonction de «l'intérêt général» de la ville et à adopter une approche « consensuelle» dans le traitement de l'information ${ }^{9}$. Cette orientation ne semble cependant pas uniforme, l'étude de Stone et Mazza n'en ayant par exemple trouvé aucune trace dans un cas précis ${ }^{10}$.

Notre analyse de la démarche journalistique s'appuie sur un examen minutieux de La Tribune et du Nouvelliste pendant la période retenue. Dans chaque municipalité, nous avons choisi les enjeux les plus percutants, ceux qui ont fait l'objet du plus grand nombre d'articles dans le journal (les autres enjeux ont aussi été étudiés, mais de façon moins systématique). Nous nous sommes également intéressés à la couverture du budget municipal, en partie parce qu'il s'agit d'une des «décisions» les plus importantes du conseil municipal et en partie parce

8. Anthony Westell, The New Society (Toronto, McClelland et Stewart, 1977).

9. Leo Bogart, «Editorial Ideals, Editorial Illusions» dans Anthony Smith (dir.), Newspapers and Democracy: International Essays on a Changing Medium (Cambridge, M.I.T. Press, 1980); Bradley Greenberg, "Community Press as Perceived by Its Editors and Readers ", Journalism Quarterly (1964): 437-440.

10. Gérald C. Stone et Patrick Mazza, «Impact of Consensus Theory on Community Newspaper Organization», Journalism Quarterly, 54 (été 1977): 313-320. 
qu'il se prête bien à l'analyse comparative. Cette analyse sera complétée par les réponses de nos informateurs à un certain nombre de questions sur la couverture que la presse parlée et écrite réserve aux affaires municipales. Seules les tendances d'ensemble seront dégagées ici. Une étude plus détaillée est présentée ailleurs ${ }^{11}$.

Il convient dès le départ d'établir le leadership du journal au niveau de l'information politique municipale. Ce leadership se manifeste de plusieurs façons. D'abord, comme on l'a vu, le journal consacre davantage de ressources que la radio et la télévision à la couverture des affaires municipales. Presque tous nos informateurs (62 sur 70) estiment que le journal parle beaucoup de la politique municipale alors qu'ils sont plus divisés lorsqu'il s'agit de la radio et de la télévision. Lorsqu'ils parlent des médias, ils pensent spontanément au journal. Plusieurs affirment que la radio et la télévision se contentent souvent de reprendre les nouvelles parues dans le journal. Finalement, à peu près tous lisent le journal quotidiennement; la consommation des autres médias est beaucoup moins régulière. Cette situation n'est pas spécifique à Sherbrooke et Trois-Rivières. Le journal local semble aussi exercer un leadership aux États-Unis et au Canada anglais ${ }^{12}$.

Ce leadership justifie la plus grande attention que nous avons accordée au journal. Mais comment caractériser la couverture qui est faite des affaires municipales? Pour ce faire, nous examinerons de près la démarche adoptée par chaque journal dans son traitement des principaux enjeux. Commençons par un enjeu commun, la présentation des budgets des années 1979, 1980 et 1981.

11. André Blais et Jean Crête, Les médias et la politique municipale dans deux villes du Québec (Québec, Laboratoire d'études politiques et administratives, Département de science politique, Université Laval, février 1982).

12. Grotta, op. cit.; Fletcher, op. cit., 31. 
Dans chaque cas, le budget fait la manchette de la page trois. Chaque fois le journal fournit des tableaux indiquant la ventilation par catégories de revenus et de dépenses, en chiffres absolus et en pourcentages. Il précise également les variations par rapport à l'année précédente du niveau de la taxe foncière et des autres taxes. Il est par contre plus silencieux en ce qui concerne les dépenses. Sauf en 1980 (et même là, l'analyse est très partielle), il ne compare pas la ventilation des dépenses à celle de l'année précédente, ce qui pourrait éclairer l'évolution des priorités du conseil. Aucune comparaison n'est établie avec d'autres municipalités de taille similaire.

Les titres sont aussi révélateurs. Pour 1979 et 1981, ils renvoient à la hausse des taxes. En 1980, les taxes ont été un peu réduites grâce à la réforme de la fiscalité municipale mais on retient l'idée de l'augmentation puisqu'à Sherbrooke le journal titre «Les dépenses franchissent le cap des $\$ 70$ millions » (La Tribune, 29 mars 1980).

Par ailleurs, seul le Nouvelliste publie, à une occasion, un article décrivant chaque étape du processus d'élaboration du budget, insistant en particulier sur le rôle central joué par le gérant et le gérant adjoint à l'administration. On ne fait à peu près pas état de réactions à la présentation du budget. En décembre 1980, La Tribune fait une petite enquête auprès de «Monsieur tout le monde» (8 individus!) le jour même de la présentation du budget, avant qu'il ne soit dévoilé pour connaître les réactions à une "majoration importante du taux de la taxe foncière qui pourrait atteindre jusqu'à $18 \%$ » et constate évidemment un mécontentement certain. On comprend difficilement l'impatience du journal qui aurait pu attendre une journée pour recueillir les opinions sur le budget réel.

Il ressort que le journal local se préoccupe hautement du niveau de taxation. Des informations précises sont données à ce sujet, en particulier en termes d'augmentation par rapport 
à l'année précédente. C'est le thème qui est retenu dans les titres. L'analyse des dépenses est par contre plus fragmentaire. Toute l'information est orientée sur la fameuse question de la hausse des taxes, qui frappe beaucoup plus l'imagination. Un exemple servira à illustrer ce point. En 1978, le taux de la taxe foncière diminue de $\$ 0.25$ à Sherbrooke. Le journal précise qu'il ne faut pas se fier aux apparences et que, suite à la hausse de l'évaluation, la diminution équivaut en réalité à une «hausse» d'environ $\$ 0.15$. On ne mentionne pas, cependant, que cette «hausse» est à peine supérieure au taux d'inflation et qu'en dollars constants, il y a donc peu de changement. De même, la baisse du taux de la taxe en 1980 ne fait pas le titre: celui-ci fait plutôt référence à la hausse des dépenses!

L'information sur le budget provient donc essentiellement de l'administration municipale. Le journal ne procède pas à une analyse plus générale ou comparative et ne fait que diffuser les données et justifications de la municipalité. Celle-ci n'exerce toutefois pas un contrôle absolu sur la nouvelle. Le journal concentre en effet son attention sur un aspect (le niveau de taxation) plus spectaculaire, l'aspect qui est le plus désagréable pour la municipalité, surtout lorsqu'il y a une hausse de taxes.

Pour le reste, il nous faut traiter séparément des deux journaux puisque leur comportement et le contexte dans lequel ils évoluent different passablement. Commençons par La Tribune. Il appert d'abord que ce journal privilégie la controverse. En cela il reflète évidemment une certaine réalité. Plusieurs controverses ont marqué la scène municipale sherbrookoise pendant la période retenue. Mentionnons en particulier le débat sur le salaire des édiles municipaux, qui s'est prolongé sur plusieurs mois et qui fit l'objet d'un référendum et la grève des employés municipaux, en décembre 1978. À cela s'ajoutent des divisions ouvertes à l'intérieur du conseil municipal sur certaines questions. 
Au départ, le journal est donc bien servi. Mais tout indique qu'il est à la recherche de la polémique et ne tente aucunement de la taire d'une façon ou d'une autre. Voici quelques indications de cette orientation. Le 29 novembre 1978, les cols bleus de la ville viennent de retourner au travail après plusieurs journées d'étude suite à une injonction interlocutoire. La veille, une importante tempête de neige, combinée au débrayage des employés municipaux, avait plus ou moins immobilisé toute la ville. La première page du journal est consacrée à ce sujet. On y retrouve même un éditorial, blâmant au passage l'administration municipale de ne pas avoir négocié sérieusement avec ses employés. Le jour même, le maire convoque une conférence de presse et accuse le journal de discréditer l'administration municipale.

Un autre exemple encore plus patent est constitué par le débat sur la direction générale. En août 1977, le directeur général de la ville remet sa démission. S'ensuit une longue discussion sur une réforme possible des structures administratives et sur le choix du nouveau directeur général. Après plusieurs échéances reportées, le nouveau directeur n'est nommé qu'en janvier 1981, après que le conseil eut opté pour la structure existante. Le journal traite abondamment de cette question, lui consacrant une quarantaine d'articles en deux ans. Le sujet prête bien à la nouvelle. Voici une controverse avec deux camps bien identifiés, tant au niveau des fonctionnaires que des membres du conseil municipal. Le journal consacre d'ailleurs un long article (3 mars 1980) à la description de ces deux groupes, identifiant clairement la position de chaque conseiller municipal. À bien des égards, l'enjeu n'était pas très substantiel mais d'un point de vue journalistique, avait l'avantage d'être caractérisé par la polémique et le suspense. La Tribune entretient d'ailleurs systématiquement le suspense comme l'attestent les titres suivants: «Une décision très bientôt» (25 mars 1979), 
«O'Bready nommera un directeur général d'ici la fin de l'année» (16 novembre 1979), «Le poste de directeur général adjoint appelé à disparaître?» (26 février 1980), «Il faut trouver une solution dans les plus brefs délais» (29 février 1980), «La question peut-être réglée le 3 juillet? » (1er juillet 1980), «Il faut se décider» (2 juillet 1980), «Nomination imminente du directeur général ?» (20 novembre 1980), «Dénouement imminent? » (28 novembre 1980), «Dénouement la semaine prochaine» ( 5 décembre 1980).

Cette recherche de la polémique crée des tensions entre l'administration municipale et la presse. Les membres du conseil municipal et les fonctionnaires sont largement insatisfaits de la couverture du journal, près de la moitié qualifiant l'information de pauvre ou très pauvre; ils se montrent plus indulgents pour la radio et la télévision. Le principal reproche adressé aux journalistes est de trop insister sur les aspects négatifs et de négliger systématiquement les aspects positifs. Seulement deux des vingt informateurs de l'administration municipale (élus et fonctionnaires) se sont dits en désaccord avec la proposition selon laquelle «les médias parlent toujours de ce qui va mal et jamais de ce qui va bien». On comprend alors assez bien pourquoi le journal est la cible de leurs critiques. Lui seul a les ressources pour fouiller certains dossiers, aller contre l'information officielle véhiculée par l'administration municipale et à l'occasion soulever certains problèmes. Les hommes politiques sont plus favorables aux médias électroniques qui ont l'avantage de leur laisser davantage de place. L'analyse y est à peu près nulle, le journaliste intervient peu, se limitant généralement à poser quelques questions.

Par ailleurs, l'initiative de l'information appartient essentiellement à l'administration municipale. Ce sont les faits, gestes et déclarations du conseil de ville qui sont à l'origine de la majorité des nouvelles publiées par le journal. La faible 
participation des groupes à la vie municipale renforce cette tendance. Le journaliste ne dispose guère d'autres sources d'information. La Tribune fait cependant des efforts pour contourner ces difficultés. Sur les enjeux où il y a division à l'intérieur du conseil municipal, le journal rapporte les points de vue différents. Dans le débat sur la direction générale, le journaliste a même contacté les conseillers avant que la question ne soit discutée au conseil; il a ainsi pu obtenir des réactions non filtrées. Le journal fait aussi état des requêtes et protestations de citoyens dès qu'elles se manifestent. Lors du référendum sur le salaire des édiles, il a fait largement état des positions de l'Association des citoyens, qui s'opposait au conseil municipal. À quelques occasions, il rapporte les requêtes de citoyens pour que des autobus passent ou (plus souvent) ne passent plus sur leur rue. Finalement, le journal a parfois recours à la petite enquête sur la rue (auprès de 5 à 10 personnes) pour donner la parole aux simples citoyens. Cette démarche est employée pour apprécier les réactions des usagers lors de la mise en application de certains changements (hausse de tarifs, modifications de trajet) dans le système de transport en commun. Ces petites enquêtes sont certes entachées de toutes sortes d'erreurs mais elles ont le mérite de fournir une autre perspective sur le service de transport en commun.

De façon générale, La Tribune se limite à présenter les différents points de vue sur une question. L'analyse y est à peu près inexistante et les positions des parties sont rarement confrontées, de sorte que le journal n'apporte guère d'éclairage original sur l'enjeu.

La couverture donnée à la grève des employés municipaux illustre bien ce point. Le traitement est essentiellement descriptif et suit le déroulement des événements: débrayage, retour au travail, demande d'injonction, assemblées du syndicat, rejet des offres, etc. Le journal rapporte les actions et 
déclarations des deux parties concernées mais ce n'est que dans quelques articles que le journal a rappelé - et brièvement la nature de l'écart qui séparait les deux parties. Aucune analyse comparative n'est faite des positions des parties si ce n'est un article qui rapporte les interprétations de la ville à ce sujet (15 décembre 1978). Aucune comparaison n'est établie avec d'autres municipalités. Le journal est systématiquement à la remorque des chiffres avancés par la ville et le syndicat, qui sont évidemment difficiles à réconcilier. Impuissant, il se plaindra de «la guerre des chiffres» (19 décembre 1978).

La conclusion de l'histoire est d'ailleurs révélatrice. Le négociateur syndical prétend avoir obtenu des augmentations salariales de près de $20 \%$ sur deux ans, qui correspondent presque exactement aux demandes syndicales (La Tribune, 4 janvier 1979, p. 1). Le maire affirme, quant à lui, que l'entente coûtera au plus $\$ 75,000$ (5 janvier 1979 , p. 4) alors qu'il avait déjà chiffré les demandes syndicales à plus de $\$ 500,000$ (15 décembre). Il y a là de quoi laisser le lecteur perplexe. Malheureusement, le journal n'a pas cru bon d'approfondir cette question mystérieuse.

Si l'analyse est si peu poussée, c'est en partie due à des contraintes de temps. Mais il faudrait aussi tenir compte de la formation des journalistes. Nous avons demandé aux journalistes s'il y avait, selon eux, certains aspects de la politique municipale auxquels les médias n'accordaient pas assez d'importance. Plusieurs ont mentionné les questions économiques et techniques (planification, réglementation), des domaines pour lesquels ils se sentent généralement moins compétents. Les journalistes semblent effectivement mal équipés pour décortiquer la portée et les implications des finances municipales ou des conventions collectives. Il en résulte une tendance à privilégier les sujets les plus controversés, qu'ils soient importants ou non. On a vu plus haut la très grande attention 
accordée au débat sur la nomination du directeur général. À l'opposé, la transaction par laquelle le Corporation municipale de transport de Sherbrooke fit l'acquisition de l'entreprise privée qui assurait le transport local jusqu'en 1978 ne mérita qu'un article (14 décembre 1978), sans qu'il n'y ait quelque analyse du prix de vente négocié. Cette question complexe et technique est jugée moins intéressante.

La Tribune, comme la plupart des médias, a tendance à négliger la mise en application des services gouvernementaux. Ce sont les grandes controverses précédant la prise de décision qui font les manchettes. Ce qui se produit par la suite, lors de l'exécution de la décision, est souvent oublié. Dans le cas de la grève des employés municipaux, par exemple, le contenu de la convention négociée n'a pas été vraiment analysé. Dans le domaine du transport, cette orientation est toutefois moins prononcée, en particulier, le journal a donné des renseignements précis sur les modalités du service et a cherché à mesurer les réactions des usagers lors de la mise en application de certains changements.

En somme, pour la période qui nous concerne, la démarche de la Tribune en est une de présentation des enjeux qui soulèvent la controverse dans le milieu. La plus grande partie des informations provient de l'administration municipale mais celles-ci sont complétées, dans la mesure du possible, par des voies moins officielles. L'analyse de ces controverses demeure superficielle.

L'approche du Nouvelliste est passablement différente. Elle est encore plus orientée vers la description des activités du conseil municipal. Cette orientation correspond au journalisme local classique, de type consensuel, s'identifiant en priorité aux normes du milieu plutôt qu'à celles du journalisme d'enquête. Le cas le plus frappant concerne la candidature de Trois-Rivières comme hôte des Jeux du Canada de 1983 (ce fut finalement 
Chicoutimi qui fut choisi). Ce dossier est celui qui, a l'exception du budget et de l'élection, fait couler le plus d'encre au Nouvelliste. Ce qui étonne l'analyste, c'est l'absence de journalisme d'enquête dans cette affaire. Le journal ne fournit aucun renseignement sur les jeux précédents. Les questions de coût et de financement sont à peine mentionnées. Aucun article ne chiffre le coût global des jeux. Tout au long du dossier, le journal se contente de rapporter fidèlement les propos du maire, ce qui donne droit à des titres comme «Le comité a choisi des jeux 'raisonnables'» (2 octobre 1979).

En conséquence, les tensions entre l'administration municipale et la presse sont quasi inexistantes. Les élus municipaux sont satisfaits de l'information publiée dans le journal. L'opinion suivante d'un conseiller résume assez bien la pensée de ses collègues: "Tout ce qui est discuté au conseil parait dans le journal». On ne formule aucun reproche de négativisme systématique. Seulement trois des élus ou fonctionnaires se sont dits d'accord avec la proposition selon laquelle «les médias parlent toujours de ce qui va mal et jamais de ce qui va bien». De même presque tous sont d'avis que les journalistes ont à cœur le développement de la ville et s'identifient à leur milieu.

Le Nouvelliste est donc très dépendant de l'administration municipale pour la génération de nouvelles. Les initiatives pour puiser à d'autres sources d'information se font très rares. Il y a bien sûr quelques exceptions. Lors d'un débat sur la fusion possible entre Trois-Rivières Ouest et Trois-Rivières, il fait faire un sondage pour connaitre les opinions des citoyens ( 7 avril 1979). Ces initiatives sont cependant timides en comparaison avec celles entreprises par $\mathrm{La}$ Tribune. Une telle orientation semble satisfaire les journalistes. Un seul informateur se montre sévère vis-à-vis la couverture offerte: "On fait du journalisme officiel. Je veux dire que c'est le maire et le conseil qui déterminent la nouvelle. Il n'y a pas de journalisme de 
recherche». Cette critique n'a été formulée que par un répondant, les autres adhérant finalement à un autre style de journalisme.

Pour le reste, les tendances observées à propos de $L a$ Tribune s'appliquent au Nouvelliste. C'est ainsi que les questions financières et techniques sont peu approfondies, que, lorsqu'il y a controverse, les positions des différents intervenants ne sont pas vraiment confrontées et que l'étape de mise en application des décisions est moins bien couverte.

Les démarches journalistiques de la Tribune et du Nouvelliste se démarquent donc assez nettement l'une de l'autre, le premier journal étant beaucoup plus orienté vers la controverse que le second. À quoi cette différence tient-elle? Le premier facteur se situe certes au niveau du conseil municipal. À Sherbrooke, l'accès à l'information est grand. Tous les journalistes interviewés estiment qu'il est facile d'obtenir de l'information des élus municipaux. La situation est différente à Trois-Rivières. Comme l'explique un journaliste, «quand on parle d'obtenir de l'information des membres du conseil à Trois-Rivières, il faut parler du maire exclusivement. Ici le maire est le porteparole». Pour ce qui est des fonctionnaires, «la plupart observent la consigne que le maire est le porte-parole».

Cela ressort clairement dans la petite analyse de contenu que nous avons faite. À Trois-Rivières, les noms des échevins ou des principaux fonctionnaires n'apparaissent que très rarement. Il en va tout autrement à Sherbrooke. Sur la question de la direction générale, par exemple, La Tribune a publié pas moins de treize articles faisant état de prises de positions de conseillers. Le journal réussit aussi à faire écho à certains remous au sein de la fonction publique.

Le niveau de centralisation de l'information conditionne donc fortement la couverture des affaires municipales. Lorsque, comme à Sherbrooke, il est faible et que les conseillers 
sont prêts à faire état de leurs divergences, les médias ont beau jeu et peuvent mettre l'accent sur la controverse, ce qui permet de faire état de certains problèmes locaux. Lorsque, comme à Trois-Rivières, l'administration décide de présenter un front uni, de ne pas ébruiter les divisions internes, il devient pour le moins difficile de fouiller des dossiers.

Ce premier facteur n'est cependant pas suffisant. On peut supposer qu'un journaliste entêté pourrait venir à bout de la consigne du silence, surtout dans le cas de conseillers particulièrement déçus d'une décision. De toute façon, les journalistes de Trois-Rivières (tant au niveau de la presse parlée que de la presse écrite) ne se préoccupent pas outre mesure de la centralisation de l'information. Presque tous jugent l'information dans Le Nouvelliste bonne ou très bonne. C'est qu'ils adhèrent à un type de journalisme plus «officiel» et consensuel, comme nous l'avons déjà indiqué.

Il apparaît donc difficile de caractériser la presse locale d'une façon ou d'une autre. Dans un cas, la politique municipale est conflictuelle et le quotidien présente effectivement une image conflictuelle de la situation. Dans l'autre cas, les élites locales ne polémiquent pas en public et le journal présente une vue consensuelle de la politique. Cette différence ne doit pas non plus faire perdre de vue plusieurs points communs. Dans les deux villes, l'essentiel de l'information origine de l'administration municipale. Dans les deux journaux l'analyse est mince, en particulier sur des questions techniques. Par contre, l'information sur les services gouvernementaux est relativement riche.

La Tribune et le Nouvelliste sont la propriété de GESCA (Power Corporation). On peut se demander si cette appartenance influe sur la démarche journalistique adoptée. Tout indique que cette influence est minime. Le contraste frappant entre les approches des deux journaux démontre que les effets de la 
concentration sont loin d'être automatiques. Un bilan plus général de la recherche au Canada n'a d'ailleurs pu déceler de différences importantes et systématiques entre les quotidiens appartenant à des chaînes et les quotidiens indépendants ${ }^{13}$. Plus spécifiquement, le processus de concentration ne semble pas avoir provoqué une diminution de la couverture des affaires municipales.

La principale différence se situe par rapport à la puissance même de l'organisation qui résulte du processus de concentration. Cette puissance a engendré un syndicalisme militant qui a obtenu pour le journaliste une grande marge de manœuvre dans la couverture des événements ${ }^{14}$. Il s'ensuit une certaine perte d'influence des élites politiques locales, qui ont probablement moins d'emprise sur la direction du journal. Cela peut favoriser un travail journalistique un peu plus fouillé et aussi plus polémique. Le cas du Nouvelliste nous rappelle toutefois que cette tendance n'est pas très prononcée.

\section{L'influence de la presse sur les politiques gouvernementales}

Si l'on s'intéresse à la presse, c'est généralement parce qu'on estime qu'il s'agit d'une institution qui exerce un impact significatif sur la société en général et la vie politique en particulier. Cet impact peut être de différents ordres. La presse peut d'abord participer à l'établissement de l'ordre du jour en aidant à déterminer les problèmes qui seront débattus en priorité sur la place publique ${ }^{15}$. À plus court terme elle peut influencer les décisions et actions précises des gouvernements.

13. Fletcher, op. cit., 135.

14. Voir aussi Lysiane Gagnon, "Journaliste et syndiqué: le perpétuel dilemme », dans Les journalistes, Montréal, Québec/Amérique, 1980, 45-71.

15. Sur cette question voir Fletcher, op. cit., 20; au niveau municipal voir David Paul Word, "The Politics of Agenda Setting in Late 19th Century Cities», Journalism Quarterly, vol. 58, 1981: 565-573. 
Nous nous limiterons ici à ce deuxième type d'impact, tout en distinguant l'impact direct lorsqu'un journal, par une prise de position (éditorial) amène l'administration municipale à adopter la voie d'action qu'elle propose (ou à abandonner une voie qu'elle dénonce) et l'impact indirect, lorsqu'un journal, en donnant une grande couverture à une question, amène l'administration à modifier une décision, à en prendre une nouvelle ou à agir avec plus de célérité.

La mesure de l'influence de la presse pose des problèmes considérables. Nous avons tout de même cherché à déterminer si, à travers les différents enjeux examinés, les prises de position du Nouvelliste et de La Tribune ainsi que leur couverture de certains enjeux ont incité le conseil municipal à modifier certaines politiques. Nous avons aussi tenu compte de la perception que nos informateurs se font de l'influence de la presse. Ces différents éléments sont autant d'indices qui, sans être complets, nous permettent de dégager certains paramètres.

Commençons par les éditoriaux. L'éditorialiste est certes un acteur important sur la scène municipale, d'autant plus que les groupes sont en général peu actifs et qu'il n'y a pas de parti politique organisé (pour la période qui nous concerne). Très rares sont les élus ou fonctionnaires qui disent n'accorder que peu d'attention aux éditoriaux. Sur les principaux enjeux considérés, toutefois, l'impact réel des éditoriaux apparaît à peu près nul. Dans Le Nouvelliste en particulier, les éditoriaux portant sur les affaires municipales sont rares. Dans les deux journaux, le budget municipal n'est généralement pas discuté en page éditoriale. On se prive ainsi de commenter ce qui constitue probablement la décision la plus importante de l'administration municipale. Les éditorialistes se montrent aussi, extrêment prudents même dans La Tribune où pourtant la couverture est beaucoup plus agressive. Lors du référendum sur le salaire des édiles, par exemple, l'éditorialiste, affirme qu'il «ne nous 
appartient pas dans le contexte actuel de nous ériger en défenseur du oui ou du non»(28 avril). Les quelques prises de position claires sont souvent tardives. C'est ainsi que le 28 août 1979, La Tribune se prononce contre l'abolition du service de transport en commun le dimanche. L'éditorial ne survient cependant que quelques jours avant la mise en application de la décision et quatre mois après l'annonce, après que de «nombreux usagers aient protesté par le biais des colonnes réservées à l'opinion des lecteurs ou par d'autres moyens». Quoiqu'il en soit, l'intervention ne fut pas fructueuse, la commission de transport ne revenant pas sur sa décision. Dans les deux municipalités, nous n'avons en somme trouvé à peu près aucune trace d'influence directe des éditoriaux sur le cours des événements.

L'influence indirecte par le biais de la couverture est encore plus difficile à évaluer. Sur les principaux enjeux analysés, le journal ne semble pas avoir eu d'impact appréciable. Une exception serait la grande publicité accordée par La Tribune à la question du salaire des édiles municipaux, publicité qui a probablement sensibilisé les citoyens à cet enjeu et indirectement favorisé le groupe de protestataires qui s'opposait au conseil municipal. Sur quelques autres points que l'on pourrait qualifier de mineurs, le journal semble avoir eu une influence. La Tribune a contribué à mettre fin aux activités du Festival des Cantons, qui se soldaient chaque année par des déficits importants. Elle a aussi réclamé avec succès la démolition du vieux stade de baseball. À l'automne 1980, les nombreux articles qu'elle a consacrés à une intersection qui était jugée dangereuse par un groupe de parents amenèrent la ville à prévoir $\$ 35,000$ pour améliorer la sécurité des piétons. À Trois-Rivières, le Nouvelliste a dénoncé la décision du conseil municipal de transformer une rue unidirectionnelle en une rue bidirectionnelle, ce qui amena le conseil à rétablir le sens unique sur cette rue marchande. Dans plusieurs de ces cas, l'éditorial vient appuyer 
un dossier déjà bien constitué pour lui donner une plus grande insistance.

Il semble donc que l'influence de la presse peut être considérable mais dans les deux villes que nous avons étudiées, elle est limitée à quelques enjeux mettant en cause la moralité, la retenue fiscale ou la mise en application de certains services à la population. Il nous apparaît d'ailleurs symptomatique que ce soit les fonctionnaires qui attribuent le plus grand impact aux medias; la très grande majorité d'entre eux estiment que les choses bougent plus vite lorsque la presse parle beaucoup d'une question. Cette conclusion concorde avec un bilan plus général de la littérature. L'influence de la presse s'exerce surtout sur des questions comportant une dimension morale ou personnelle. De même, plusieurs rédacteurs en chef de petits quotidiens «ont affirmé que leurs efforts avaient empêché des augmentations de traitement injustifiées pour les politiciens municipaux ou aidé à éviter des majorations d'impôt » ${ }^{\mathbf{1 6}}$.

L'influence s'exerçant plutôt de façon indirecte, par la publicité accordée à un enjeu, le journal qui fouille de façon plus indépendante les différents dossiers risque d'avoir plus de pouvoir. C'est pourquoi l'impact de La Tribune apparaît globalement plus important que celui du Nouvelliste. Cette association n'est cependant pas sans contre-tendance. C'est ainsi qu'à Sherbrooke les élus ont tendance à se tourner un peu plus vers les medias électroniques, parce que, selon certains informateurs, ils s'estiment mieux couverts et que la presse électronique est moins critique envers eux. Le fait qu'une telle réaction ne semble pas se manifester à Trois-Rivières, où le journal est peu critiqué, accrédite cette thèse. Les hommes politiques utiliseraient ainsi à leur avantage la compétition entre les medias. Cela suggère une autre limite au pouvoir du jour-

16. Fletcher, op. cit., 27. 
nal. Ce pouvoir réside surtout dans l'analyse critique mais si cette critique se fait trop virulente, les élus favoriseront d'autres medias plus «sympathiques».

\section{La presse et l'élection municipale}

L'on ne saurait traiter du rôle de la presse dans les affaires municipales sans s'intéresser à son comportement lors des élections municipales, l'élection étant à la base même de notre système de représentation. Dans les deux municipalités une élection fut tenue en novembre 1978. À Sherbrooke le maire et trois conseillers furent élus par acclamation; la campagne électorale s'est limitée à sept quartiers. À Trois-Rivières, il y eut élection à la mairie et à tous les sièges. L'élection des conseillers se faisait sur l'ensemble du territoire et non par quartier, comme à Sherbrooke.

Dans les deux municipalités, la couverture débute par l'annonce des candidatures. Le journal présente en trois ou quatre paragraphes le curriculum vitae du candidat et les priorités qu'il met de l'avant. Pour les membres du conseil qui désirent renouveler leur mandat, on rappelle les dossiers qu'ils ont pilotés.

Quelques jours avant la présentation officielle des candidatures, le Nouvelliste dresse un tableau de la situation à chaque siège. Un seul commentaire accompagne ce tour d'horizon rapide, traitant par exemple du nombre d'années de présence au conseil d'un échevin sortant ou des tentatives antérieures d'un nouveau candidat. La Tribune, elle, publie une série de trois articles intitulés «la parole aux candidats» $(25,27$ et 28 octobre 1978). Le journaliste a posé à tous les candidats déclarés des questions identiques sur un certain nombre de sujets. Les réponses de chacun sont rapportées brièvement, en quelques lignes. 
Pendant la dernière semaine de la campagne, les articles se font très rares. À Sherbrooke, aucune assemblée publique ne semble avoir lieu. À peu près rien ne semble se prêter à la nouvelle. Les quelques exceptions sont révélatrices. On fait état de la demande d'un débat public par un candidat et du refus de son adversaire. Un court filet rapporte la déclaration du seul candidat féminin à l'effet que sa condition de femme était un atout pour le scrutin. Un autre article, plus important, traite de la déclaration d'un échevin identifié comme étant le leader d'un des deux clans au conseil de ville, reconnaissant l'existence de ces clans et ajoutant qu'il ne cherchait pas à prendre le pouvoir au conseil... pour l'instant! Cette bataille des clans fait évidemment une bonne nouvelle.

La situation n'est guère différente à Trois-Rivières. L'événement marquant de la dernière semaine fut une assemblée publique organisée par la Chambre de commerce et réunissant tous les candidats. L'événement est bien couvert par le Nouvelliste qui y consacre deux articles, le premier s'attachant au déroulement de l'assemblée et le second, plus analytique, en dressant un bilan. Un seul paragraphe est réservé aux aspirants-conseillers, qui conclut qu'aucun d'entre eux n'a réussi à impressionner. On n'y retrouve rien qui puisse éclairer la lanterne du citoyenélecteur.

Les candidats à la marie retiennent plus l'attention. Le journal mentionne que le maire sortant tente «avec sérieux et dignité de défendre ses réalisations» sans toutefois préciser le contenu de ses propos. Quant aux deux autres candidats, le journal souligne surtout leur manque de crédibilité, l'un étant désigné comme «le candidat lutteur-chanteur-chômeur», brandissant «tantinet de doctrine créditiste » en promettant du travail pour tous et l'autre, demeurant «fidèle à lui même en portant à bout de bras la cause de la veuve et de l'orphelin» et cherchant à «faire sortir le vote des athées de la ville». 
Aucun autre article ne parut pendant la dernière semaine sur la campagne électorale à Trois-Rivières. Le Nouvelliste n'a fourni aucune information sur les positions des candidats sur les principaux enjeux.

Cette couverture électorale appelle un certain nombre de commentaires. L'absence de lutte véritable à la mairie et d'un débat de fond sur l'orientation de la politique municipale laissait peu de prise aux journaux. Face à cette situation La Tribune a tout de même réussi à présenter les positions des candidats échevins sur certaines questions. L'initiative est fort intéressante. Elle a bien sûr des limites. Pour des raisons inconnues, les entrevues ont été effectuées avant la fin de la période de nomination, de sorte que deux candidats de la dernière heure n'ont pu être interviewés. Le journaliste n'a pas comparé systématiquement sur les différentes questions les candidats qui se font la lutte. Cette série de trois articles n'en a pas moins permis aux lecteurs de situer les candidats sur un certain nombre d'enjeux.

L'information diffusée par le Nouvelliste fut encore plus pauvre. Sa tâche était rendue difficile par le manque de crédibilité des adversaires du maire. Mais le journal n'a aucunement réussi à dégager les enjeux réels ou potentiels par rapport auxquels les aspirants-conseillers pouvaient se situer.

La couverture de la campagne électorale fut donc assez mince. Cette situation ne semble pas spécifique aux deux villes étudiées, la même tendance ayant été observée ailleurs ${ }^{17}$. Malgré cela, la plupart de nos informateurs se sont dits satisfaits de la couverture offerte par les medias. La très grande majorité des hommes politiques reconnaissent que la presse a fait preuve d'équité et a traité à peu près également tous les candidats. Chez les journalistes, on avait le sentiment qu'il n'y avait pas

17. Fletcher, op. cit., 115. 
de véritable enjeu, que la campagne se résumait à des querelles de personnalités ou de clochers et que les médias ont fait ce qu'ils ont pu dans les circonstances. Cette position peut se défendre dans le cas de La Tribune, qui a présenté une analyse comparative des candidats mais nous semble difficilement soutenable en ce qui concerne Le Nouvelliste, dont la couverture a été extrêmement pauvre.

Par contre, les candidats battus lors de cette élection se sont dits insatisfaits de la couverture, surtout les nouveaux candidats, les quelques conseillers sortant défaits étant plutôt satisfaits. Ils se plaignent surtout de leur incapacité à faire connâ̂tre leurs positions dans les médias autrement que par la publicité payée. À la décharge des médias, il faut admettre que l'absence apparente d'enjeu central rendait leur tâche difficile. La réforme de la démocratie municipale et l'implantation de partis politiques devraient donner naissance à des campagnes plus animées et, espérons-le, à une couverture plus attentive. Quoi qu'il en soit, l'absence de véritable couverture pénalise davantage les nouveaux candidats moins connus et contribue indirectement à la réélection des conseillers sortants.

Un reproche spécifique mérite d'être souligné. Quelques hommes politiques prétendent que les médias désirent obtenir de la publicité payée de la part des candidats et pour cette raison ne donnent que très peu d'information sur les positions des candidats. Nous ne sommes pas en mesure de déterminer si tel est effectivement l'objectif poursuivi mais nous ne pouvons que constater la pauvreté globale de la couverture. Les articles sur la campagne électorale se font étonnamment rares dans la dernière semaine de la campagne.

Par ailleurs, les deux journaux se sont abstenus d'appuyer officiellement quelque candidat en page éditoriale, quoique Le Nouvelliste n'hésite pas à qualifier les deux adversaires du maire de «fantaisistes». Ce comportement n'est pas surprenant. 
Dans une enquête portant sur 64 quotidiens au Canada, on a noté d'une part qu'un "plus grand nombre de quotidiens se sont abstenus de tout commentaire éditorial pendant les élections locales que lors des élections fédérales et provinciales», et d'autre part, que plusieurs rédacteurs en chef estiment que l'appui d'un quotidien jouissant d'un monopole est injuste ${ }^{\mathbf{1 8}}$. Ce faisant, le journal se conforme à la norme du milieu. Seulement cinq de nos informateurs sont d'avis que le journal devrait appuyer des candidats lors d'une élection. Plusieurs ont justifié leur position par le pouvoir démesuré du journal face à tout candidat. Cela constitue une autre limite au pouvoir du journal. La norme de la neutralité n'est cependant pas absolue. Plusieurs informateurs reconnaissent au journal le droit de se prononcer dans des circonstances exceptionnelles, par rapport à des candidatures loufoques par exemple. On a vu que Le Nouvelliste s'est prévalu de cette possibilité.

En somme, comme pour les autres enjeux, l'influence de la presse sur le résultat de l'élection est indirecte. Les éditorialistes se montrent extrêmement prudents et n'osent pas appuyer ouvertement un candidat. La lutte au niveau de l'échevinage ne leur laisse que peu de prise, d'autant plus qu'ils ne se donnent pas les moyens pour dégager certains problèmes latents. Le principal impact des médias se situe probablement au niveau de ce qu'ils ne font pas. En ne couvrant que faiblement la campagne, ils favorisent involontairement la réélection du conseil sortant.

\section{Conclusions}

Dans le processus d'information sur les affaires municipales, il est clair que l'initiative appartient aux élus et aux fonctionnaires, la presse n'étant souvent qu'une courroie de transmis-

18. Fletcher, op. cit., 98-101. 
sion. Si la politique municipale est faite de contestations et d'oppositions, le journal tend à être polémique. Si elle est faite d'accords et d'agréments, il tend à être consensuel. En transmettant l'information, cependant, le journal filtre ou amplifie. La Tribune recherche davantage la controverse et le sensationnalisme et Le Nouvelliste s'oriente dans les campagnes menées «au bénéfice de la communauté régionale ${ }^{19}$. Par ailleurs, l'influence de la presse apparaît potentiellement très grande mais ne semble s'exercer concrètement que dans des enjeux mettant en cause la moralité, la fiscalité ou des services concrets à la population.

Le journalisme local apparaît ainsi tiraillé entre deux grandes tendances. La première est la pratique de la presse nationale, qui incite à privilégier la primeur, le sensationnel et la controverse. La seconde est l'identification au milieu, qui incite à rapporter les faits et gestes de l'élite et à appuyer celleci dans ses revendications auprès des gouvernements provincial et fédéral. Chaque journal se fait une niche entre ces deux tendances.

D'aucuns conclueront que la qualité de l'information est encore plus pauvre dans la presse locale que dans la presse nationale. Dans la mesure où la qualité est supposée reposer sur un journalisme d'enquête, ils auront raison. Un tel type de journalisme est à peu près inexistant à Trois-Rivières et même à Sherbrooke. L'écart avec la presse nationale est cependant moins considérable qu'on pourrait le croire. Certes l'esprit «critique» est beaucoup plus développé dans la "grande presse», où l'on aime bien tenir le rôle du rebelle qui s'oppose à l'ordre établi ${ }^{20}$. L'esprit critique par contre, n'a que peu de portée

19. Bertrand Tremblay, "Grandeurs et misères de la presse régionale » dans Florian Sauvageau et al. (dir.), Dans les coulisses de l'information: les journalistes (Montréal, Québec-Amérique, 1980).

20. Robert Fulford et al., Du côté des journalistes (Ottawa, Commission royale sur les quotidiens, 1981). 
s'il n'est pas orienté sur des questions substantielles. Lors des élections fédérales de 1979 et 1980, par exemple, les médias ont critiqué vivement les stratégies des partis, ce qui les a amenés à consacrer beaucoup d'énergie au déroulement de la campagne et à négliger les positions (ou absences de position) des partis sur des dossiers importants ${ }^{21}$. Il n'est pas évident qu'une analyse très fine des stratégies électorales contribue davantage à l'information du citoyen qu'un compte-rendu des décisions du conseil municipal.

Il est donc grand temps de se départir d'une certaine condescendance vis-à-vis la presse locale. Alors que le tirage des quotidiens québécois a diminué sensiblement entre 1970 et 1980, celui de La Tribune et du Nouvelliste a augmenté légèrement $^{22}$. La nouvelle locale et régionale est celle qui intéresse le plus le lecteur canadien; ce même lecteur trouve que la plus grande lacune des quotidiens c'est le manque de nouvelles loca$\operatorname{les}^{23}$. Finalement la seule dimension où la presse écrite, surclasse les médias électroniques est dans son rôle de service à la communauté locale ${ }^{24}$. La presse locale est importante, tout autant que la presse nationale. Elle a certaines caractéristiques propres. Les journalistes sont habituellement jeunes ${ }^{25}$, peu spécialisés et disposent probablement de moins de ressources pour résister à l'influence des élites politiques. Les normes journalistiques ne sont pas tout-à-fait les mêmes. Cela ne fait que confirmer la nécessité d'une analyse systématique de cette presse moins «prestigieuse» mais bien vivante.

21. Fletcher, op. cit., 107.

22. Léonard Kubas et le Communications Research Center, Les quotidiens et leurs lecteurs (Ottawa, Commission royale sur les quotidiens, 1981).

23. Ibid., 71 et 37.

24. Ibid., 27.

25. Roger de La Garde, "Profil sociodémographique des journalistes de la presse écrite québécoise», Communication et information, vol. 1, 1975, 37; Fulfort, op. cit., 63. 